https://doi.org/10.48009/1_iis_2006_114-118

\title{
COMPUTER-ASSISTED INSTRUCTION VS. TRADITIONAL INSTRUCTION IN AN ADVANCED-LEVEL COMPUTER COURSE
}

\author{
J. D. Thomerson, Valdosta State University, jthomers@valdosta.edu
}

\begin{abstract}
The purpose of this study was to compare student perceptions of computer-assisted instruction versus traditional instruction in an advanced-level computer applications course. The computer-assisted instruction that was used was an audio/video screen capture software program called HyperCam. The study used a survey instrument containing Likertscale statements and several open-ended questions dealing with a variety of issues related to computer instruction and the computer-assisted software program. The survey was administered to several different groups of students over a two-year period with an overall $N$ of 83 . Results of the study indicated that students rated both methods of instruction very favorably with a slight tendency to favor the CAI video lesson instruction. Students overwhelmingly favored a balanced mix of CAI video lesson instruction and traditional instruction.
\end{abstract}

Keywords: Computer-Based Instruction, Multimedia, Information Technology, Distance Education

\section{INTRODUCTION}

Technology has changed the way business, industry, educational environments, and the government conducts training. The National Center for Education Statistics reported dramatic increases in the number of on-line and web-based training courses being offered by post-secondary institutions [2]. Unfortunately, they also report a high percentage of these courses use low-level interaction techniques due primarily to the fact that "text-based" communication is easy and the most compatible with existing software. Kruse and Keil [1] stated:

"There are only two real disadvantages to WebBased Training (WBT) and both will be overcome in the next five to ten years as high bandwidth network connection become as common as telephones. The first drawback of WBT compared to live instruction is the lack of human contact, which greatly impacts learning...The second major drawback is the lack of multimedia in many WBT programs. The use of audio and video are critical to creating compelling metaphors and realistic job simulations" [1, p. 55].

There are numerous multimedia tools available today, Year 2006, that can provide the needed video, sound, animated graphics, and text needed to overcome the two limitations mentioned by Kruse and Keil [1]. Many of these software programs, such as HyperCam, are comparatively inexpensive and easy to learn. Network capabilities today can also better handle the large amounts of data that once limited computer based training.

\section{Audio/Video Screen Capture Using HyperCam}

All teachers who have taught a hands-on computer course know the value of simple step-by-step directions. These directions usually come either in the form of teacher-led computer demonstrations using a projector or television monitor hooked to the computer or as a tutorial in a computer textbook. Textbook tutorials can often be poorly written and hard to follow. Students often skip a step in the textbook and get confused with the directions. In addition, a form of "monkey see monkey do" often occurs. The students rush through the tutorial and finish, but they have not really learned anything. Teacher-led demonstrations of computer software are often a better option. However, this is a one-time event. Few teachers are willing to present the entire presentation again if a student got a bit lost, forgot some of the material, or was absent the day the presentation was made. Technology available today makes it possible for the teacher to capture these onscreen computer presentations as files that can be viewed repeatedly by students. Students can watch these presentations on their computer screens and fast-forward, rewind, and pause the presentation as needed.

The type of computer software used to record these on-screen computer presentations is called video capture software. These software packages capture the action from your screen and saves the presentation as an AVI (Audio-Video Interleaved) movie file. In addition, through the use of a microphone, the teacher can record his/her voice explaining what is occurring on the screen. These files can be loaded and viewed in any media player 
such as Real Player or Windows Media Player. HyperCam, is a very popular screen capture software package that is extremely effective, easy to use, and inexpensive.

\section{Purpose and Objectives}

The purpose of this study was to compare the perceptions of the computer-assisted instruction (CAI) audio/video lesson presentation approach using HyperCam versus the traditional (lecture/demo/work together) presentation approach of learning new computer skills in an advanced-level, hands-on computer course. Demographic factors that might affect these perceptions were also gathered.

\section{Methods}

To obtain this information, a survey instrument was developed. Numerous prior distance education survey instruments were examined to help write valid and reliable questionnaire items. However, due to the unique nature of this research, all the questionnaire items were original. Two colleagues and a graduate teaching assistant familiar with the CAI video lessons helped edit the instrument for clarity, comprehensiveness, and grammar. This survey instrument was administered to two advanced-level computer classes during the Spring 2003 Semester, to one advanced-level computer class during Fall 2003 Semester, and to two additional advanced-level classes during the Spring 2004 Semester, making an overall $\mathrm{N}$ of 83 . These classes and students represented all available students exposed to the CAI video lesson teaching approach during this time period.
All classes were taught by the same instructor and thus the CAI video lessons and the traditional class demonstrations were created and/or presented by the same person. The students attending these classes were exposed to approximately $50 \%$ traditional instruction and 50\% CAI video instruction during the course. This was accomplished by simply alternating weeks or units of study between the traditional presentation approach and the CAI video lesson approach. All students were exposed to the same presentation approach depending on what approach was chosen to be used for a particular unit of study. Students in all classes completed the survey instruments the last regular class day of the semester. Every student enrolled in each course completed the survey.

\section{Findings/Results}

The survey instrument was divided into four sections dealing with student perceptions of the CAI instruction and the traditional instruction they received in the advanced computer course. Section A dealt with the learning effectiveness of both methods; Section B asked students to rate their preferences for various aspects of the class and the two instructional approaches; Section $C$ dealt with the advantages and disadvantages of both methods; and Section D had students rate in $1,2,3$ order their preference of learning new computer concepts using the CAI approach, the traditional approach, or a $50 \%$ blend of both approaches. Results of each of these sections are shown in Table 1.

Table 1. Sections A, B, C, and D of Survey Instrument

\section{Section A:}

Students were asked to rate the learning effectiveness of both instructional approaches by checking the appropriate box on the survey instrument. Results (in percentages) are shown below.

CAI Video lesson

Traditional lecture/demo/ work together

$\begin{array}{ccc}\text { Very Effective } & \text { Effective } \\ 54 \% & 44 \% & \begin{array}{c}\text { Ineffective } \\ 2 \%\end{array} \\ 43 \% & 52 \% & 5 \%\end{array}$

Very Ineffective
$0 \%$
$0 \%$




\section{Section B:}

Students were asked to rate by checking the appropriate box their level of agreement or disagreement with the following statements. Results (in percentages) are shown below.

I prefer CAI video lessons over the traditional presentation approach

I found the CAI video lessons to be easy to follow and understand.

I found the traditional presentation format to be easy to follow and understand.

I prefer the traditional presentation approach of instruction over the CAI video lessons.

I retained information better when instruction was delivered via the traditional presentation approach.

I retained information better when instruction was delivered via the CAI video lesson approach.

I dislike the CAI video lesson approach.

I dislike the traditional presentation approach.

The use of CAI video lessons improved my performance in this course.

The use of the traditional presentation approach improved my performance in this course.

I was more motivated to learn new material when using the CAI video lessons as opposed to the traditional presentation approach.

I was more motivated to learn new material when using the traditional presentation approach as opposed to the CAI video lessons.

The traditional presentations were boring.

The CAI video lessons were boring.

\begin{tabular}{|c|c|c|c|}
\hline Strongly Agree & Agree & Disagree & Strongly Disagree \\
\hline $39 \%$ & $30 \%$ & $22 \%$ & $9 \%$ \\
\hline $54 \%$ & $35 \%$ & $11 \%$ & $0 \%$ \\
\hline $42 \%$ & $54 \%$ & $4 \%$ & $0 \%$ \\
\hline $20 \%$ & $26 \%$ & $46 \%$ & $8 \%$ \\
\hline $24 \%$ & $28 \%$ & $41 \%$ & $7 \%$ \\
\hline $28 \%$ & $37 \%$ & $28 \%$ & $7 \%$ \\
\hline $13 \%$ & $7 \%$ & $42 \%$ & $38 \%$ \\
\hline $0 \%$ & $13 \%$ & $63 \%$ & $24 \%$ \\
\hline $28 \%$ & $46 \%$ & $22 \%$ & $4 \%$ \\
\hline $26 \%$ & $39 \%$ & $33 \%$ & $2 \%$ \\
\hline $29 \%$ & $27 \%$ & $40 \%$ & $4 \%$ \\
\hline $18 \%$ & $22 \%$ & $53 \%$ & $7 \%$ \\
\hline $7 \%$ & $20 \%$ & $67 \%$ & $6 \%$ \\
\hline $6 \%$ & $20 \%$ & $52 \%$ & $22 \%$ \\
\hline
\end{tabular}


The classroom experience was more enjoyable on days when CAI video lessons were used as opposed to traditional presentations.

The classroom experience was more enjoyable on days when the traditional presentation approach was used as opposed to CAI video lessons.

I enjoyed being a part of a new instructional approach such as the CAI video lessons.

\section{Strongly Agree Agree Disagree Strongly Disagree}

$33 \%$

$43 \%$

$4 \%$

$9 \%$

$30 \%$

$50 \%$

$11 \%$

$37 \%$

$41 \%$

$20 \%$

$2 \%$

\section{Section C:}

The following two tables report (in percentages) student perceptions of the advantages and disadvantages of the two learning approaches used in the advanced-level computer course. Students were instructed to check all that apply.

\section{Advantages of CAI video lessons}

$\underline{89 \%}$ ability to review work when absent

$\underline{26 \%}$ more instructor attention

$\underline{87 \%}$ ability to work at one's own pace

$\underline{85 \%}$ ability to review instruction as needed

$\underline{85 \%}$ ability to pause instruction

$\underline{52 \%}$ less time consuming

\section{Advantages of traditional presentation approach}

$\underline{80 \%}$ ability to ask questions

59\% "live" instruction

$\underline{43 \%}$ clearer explanations

$\underline{46 \%}$ motivation from instructor

$\underline{43 \%}$ more instructor attention

\section{Disadvantages of CAI video lessons}

$\underline{30 \%}$ boring

$\underline{43 \%}$ lack of "live" instruction

$\underline{28 \%}$ difficulty comprehending information from screen $\underline{30 \%}$ difficulty hearing

$22 \%$ must be self-motivated

\section{Disadvantages of traditional presentation approach}

$33 \%$ boring

$\underline{57 \%}$ class must stay in "lock-step"

$\underline{74 \%}$ more time consuming

$\underline{39 \%}$ class interruptions

$\underline{48 \%}$ inability to review instruction

$\underline{54 \%}$ inability to pause to take notes

\section{Section D:}

The table below shows the order of preference of learning new computer concepts in the ACED 3400 course. Results are shown in percentages.

\begin{tabular}{|l|c|c|c|}
\cline { 2 - 4 } \multicolumn{1}{c|}{} & $\mathbf{1}^{\text {st }}$ choice & $\mathbf{2}^{\text {nd }}$ Choice & $\mathbf{3}^{\text {rd }}$ Choice \\
\hline $100 \%$ CAI video lessons & $33 \%$ & $26 \%$ & $42 \%$ \\
\hline $\begin{array}{l}50 \% \text { CAI video lessons } / 50 \% \text { traditional } \\
\text { presentations }\end{array}$ & $47 \%$ & $53 \%$ & $0 \%$ \\
\hline $100 \%$ traditional presentations & $21 \%$ & $21 \%$ & $58 \%$ \\
\hline
\end{tabular}




\section{Demographic Data}

The students in the survey were $54 \%$ female and $46 \%$ male. Seventy-two percent of the students were between the ages of 18 and 22; fifteen percent between the ages of 23 and 25; and thirteen percent were age 26 or above. Student classification was as follows: $6 \%$ freshman; $26 \%$ sophomore; $32 \%$ junior; $28 \%$ senior; and $8 \%$ graduate. Ninety-one percent of students reported that they had never previously had a class that used a computer-assisted instruction presentation format. All of the students reported having had previously taken at least one hands-on computer application course in either high school or at the post-secondary level and $73 \%$ of the students reported that they had taken three or more computer courses.

\section{CONCLUSIONS}

The results of this survey indicate that students liked both the traditional teaching approach and the CAI video lesson approach to instruction with a slight tendency to favor the CAI Video Lesson format. Students stated that they learned new material effectively using either method. Although students slightly favored CAI instruction, it is interesting to note that most did not want 100 percent CAI instruction. Also, it is worth noticing that the lowest rated teaching method was the $100 \%$ traditional approach.
Based upon the results of this survey it can be argued that the advantages of incorporating computerassisted instruction outweigh the disadvantages. In particular, advantages such as the ability to review work (especially when absent), ability to work at one's own pace, and the ability to pause instruction were viewed highly favorable by students. The ability to ask questions during instruction was the highest rated advantage of the traditional teaching approach. Although there were disadvantages of the CAI video lessons these disadvantages were not rated very high especially when compared with the disadvantages of only using the traditional teaching method.

A recommendation to computer instructors is to use a variety of instructional methods-students do not wish 100 percent CAI instruction much as they do not wish 100 percent lecture. This study has indicated that CAI Video instruction is an effective teaching method for advanced computer classes.

\section{REFERENCES}

1. Kruse, K. \& Keil, J. (2000). Technology-based training: The art and science of design, development, and delivery. San Francisco, CA: Jossey-Bass/Pfeiffer.

2. NCES. (2002). National Center for Education Statistics. Available

http://www.nces.ed.gov/surveys. 